\title{
The impact of a vaginal brachytherapy boost to pelvic radiation in stage III endometrial cancer
}

\author{
Adam Huddleston, MD!, Sally Zhen, MD!, Lihong Qi, PhD², Dominique Rash, MD!, Gary Leiserowitz, MD³, \\ Jyoti Mayadev, MD' \\ 'Department of Radiation Oncology, UC Davis Cancer Center, University of California Davis, Sacramento, CA, ${ }^{2}$ Department of Public Health \\ Sciences, Division of Biostatics, University of California Davis, Sacramento, CA, ${ }^{3}$ Department of Gynecology Oncology, University of California \\ Davis, Sacramento, CA, USA
}

\begin{abstract}
Purpose: We investigate the use and impact of a vaginal brachytherapy boost (VBB) after pelvic radiotherapy for stage III endometrial adenocarcinoma on vaginal and pelvic control.

Material and methods: One hundred patients treated from 1998-2011 with surgery and adjuvant therapy with or without a VBB were included. Variables examined were grade, stage, lymphovascular space invasion (LVSI), vaginal involvement (VI), cervical stromal involvement (CSI), myometrial invasion (MI), and a VBB. Failure was scored as vaginal, or pelvic. Fisher's exact test assessed association between variables with vaginal and pelvic control.

Results: With a median follow up of 43 months, $31 \%$ were stage IIIA, $6 \%$ stage IIIB, and $63 \%$ stage IIIC. Thirty-eight $(38 \%)$ received pelvic radiotherapy alone, and $62 \%$ received adjuvant chemotherapy. Of the 100 patients, 82 were treated with a VBB, 10 were not treated with a VBB, and 8 were not treated with RT. Of the 82 patients who received a VBB, 5 failed in the vagina with vaginal and pelvic control rates of $94 \%$ and $92 \%$. The impact of VB reached borderline significance with its impact on pelvic control, $92 \%$ vs. $70 \%(p=0.056)$, and did not affect vaginal control, $94 \%$ and $90 \%$ $(p=0.50)$. Neither tumor grade, LVSI, CSI, stage, nor LVSI $(p>0.05)$ statistically significantly impacted vaginal control.

Conclusions: There are no clinical guidelines for the use of a VBB in stage III endometrial cancer. The majority of our patients were treated with a VBB and experienced excellent pelvic and vaginal control. The presence of traditional adverse features did not negatively impact control in our patient cohort. However, the role of a VBB needs further investigation to understand the incremental benefit beyond pelvic RT.
\end{abstract}

Key words: endometrial cancer, pelvic radiation therapy, vaginal brachytherapy.

\section{Purpose}

Endometrial cancer is the most common female malignancy of gynecologic origin with an estimated incidence of 47,130 new cases and 8,010 deaths in 2012 [1]. Unfortunately, there has been little change in 5-year overall survival rates since the mid 1970's. The management of endometrial cancer therefore continues to evolve with refinement of both surgical approaches and adjuvant therapy. Surgery remains the indisputable standard of care for initial management of uterine disease. Various approaches continue to be utilized for management of locally advanced endometrial cancer, especially for women with regional lymph node involvement. It has been well established that women with FIGO stage III endometrial cancer benefit from adjuvant therapy in the form of chemotherapy and radiotherapy to improve local control, disease free survival and overall survival outcomes [2-6]. Often a matter of institutional preference, these modalities are used independently or in some sequential combination, as both have shown benefit albeit with continual debate surrounding ideal order and timing of these therapies [6].

An unanswered question is whether women with stage III disease who receive whole pelvis radiation therapy (WPRT) as part of their adjuvant therapy derive meaningful benefit from the addition of a vaginal brachytherapy boost (VBB). Currently, there is limited data addressing the need for vaginal brachytherapy as a compliment to pelvic radiotherapy or systemic chemotherapy in stage III endometrial cancer patients. This investigation examines the impact of a VBB on disease control, using patients treated with adjuvant WPRT with or without vaginal brachytherapy following optimal surgery for International Federation of Obstetrics and Gynecology (FIGO) stage III endometrial cancer. Currently, there are no guidelines for clinicians on when to consider a VBB. Our primary study endpoints include the impact 
of a VBB on pelvic and vaginal control. We also explore the risk factors associated with the selection of a VBB.

\section{Material and methods}

The institutional tumor registry and departmental databases were queried to identify patients who were consecutively and definitively treated for FIGO stage III endometrial adenocarcinoma, clear cell or serous cancer between 1998 and 2012. The Institutional Review Board granted permission for this study. Patient characteristics considered included age, race, Karnofsky performance status, ethnicity, and medical comorbidities including the presence of diabetes and hypertension. The pathological tumor characteristics included FIGO stage, degree of myometrial invasion, cervical stromal involvement, lymphovascular space invasion, grade, adnexal and vaginal involvement, and status of pelvic and paraaortic lymph nodes. Treatment characteristics included type and number of chemotherapy cycles given, external beam dose, treatment modality, and vaginal brachytherapy dose. Outcome measures were local control, vaginal, and pelvic control.

A total of 100 consecutive patients were treated from 1998 to 2012 were included in the analysis. All patients underwent a total abdominal, laproscopic, or robotic assisted hysterectomy, and bilateral salpingo-oophorectomy with either lymphadenectomy or lymph node sampling, peritoneal washings and omental biopsy. Patients had their pathology reviewed by a specialist in the field of gynecological cancer. One patient was treated preoperatively and was subsequently lost to follow-up. Descriptive analyses were performed to characterize the clinical, demographic, and pathological features of the patient population. Assessed variables include performance status, tumor grade, pathologic $\mathrm{T}$ stage, $\mathrm{N}$ stage, lymphovascular space invasion (LVSI), vaginal involvement (VI), lower uterine segment invasion (LUS), cervical stromal involvement (CSI), myometrial invasion (MI), and use of a VBB. Failure was scored as vaginal, pelvic, abdominal or distant. Fisher's exact tests were used to assess the association between these variables on vaginal and pelvic control.

\section{Results}

\section{Patient characteristics}

As shown in Table 1, of the 100 patients, $13 \%$ were Latino, $43 \%$ white, and $8 \%$ black. Also $69 \%$ had a Karnofsky performance status of 90 or 100 . In terms of medical comorbidity, $48 \%$ has diabetes and $52 \%$ had hypertension. Table 2 presents the tumor characteristics of the patients. The stages of the patients were 31 stage IIIA, 6 stage IIIB, 39 stage IIIC1, and 24 stage IIIC2. In addition, $77 \%$ of the population was adenocarcinoma and $57 \%$ had LVSI, 30\% had adenexal involvement, $43 \%$ had LUS involvement, 31\% CSI, and 8\% had VI as shown in Table 2 .

\section{Treatment}

Of the 100 patients, 82 were treated with WPRT and a VBB, 10 were treated with WPRT alone (no VBB), and
8 were not treated with RT. In the analysis, 92 patients were treated with whole pelvic radiation for a total dose of 45-50.4 Gy in 1.8-2 Gy per fraction. A single patient was treated with whole abdominal radiotherapy to a dose of $30 \mathrm{~Gy}$ followed by a boost of $19.8 \mathrm{~Gy}$ to the pelvis alone. The average elapsed time for the external beam was 39 days. Three dimensional conformal radiation therapy was the primary method of external beam radiotherapy with the use of intensity modulated radiation therapy increasing over the course of the study period. Sixty-two percent of patients were also treated with systemic chemotherapy, the majority with carboplatin and paclitaxel for an average of 5 cycles, while cisplatin as a single agent was used in 3 patients.

Among all patients, 82 patients were treated with a VBB using a high dose rate Ir-192 afterloader. Brachytherapy was delivered to the upper one-third to one-half of the vagina and was prescribed to either the surface

Table 1. Patient characteristics

Patients $(n) \quad 100$

\begin{tabular}{|c|c|}
\hline \multicolumn{2}{|l|}{ Ethnicity } \\
\hline Hispanic or Latino & 13 \\
\hline Not Hispanic or Latino & 70 \\
\hline Unknown/Not reported & 17 \\
\hline \multicolumn{2}{|l|}{ Race } \\
\hline American Indian/Alaska Native & 0 \\
\hline Asian & 6 \\
\hline Native Hawaiian or other Pacific Islander & 3 \\
\hline Black or African American & 8 \\
\hline White & 43 \\
\hline More than one race & 0 \\
\hline Unknown/Not reported & 40 \\
\hline \multicolumn{2}{|l|}{ Performance Status } \\
\hline 50 & 1 \\
\hline 60 & 3 \\
\hline 70 & 10 \\
\hline 80 & 14 \\
\hline 90 & 52 \\
\hline 100 & 17 \\
\hline \multicolumn{2}{|l|}{ Diabetes } \\
\hline Yes & 48 \\
\hline No & 52 \\
\hline \multicolumn{2}{|l|}{ Hypertension } \\
\hline Yes & 52 \\
\hline No & 48 \\
\hline
\end{tabular}


Table 2. Tumor characteristics

Tumor characteristics

Patient number

\begin{tabular}{ll}
\hline Patient pathologic stage (FIGO) & \\
\hline IIIA & 31 \\
\hline IIIC1 & 6 \\
\hline IIIC2 & 39 \\
\hline Patient pathologic T stage (AJCC) & 24 \\
\hline T1a & 16 \\
\hline T1b & 27 \\
\hline T2 & 9 \\
\hline T3a & 35 \\
\hline T3b & 13
\end{tabular}

Patient pathologic $N$ stage

\begin{tabular}{ll}
\hline NO & 38 \\
\hline N1 & 37 \\
\hline N2 & 25
\end{tabular}

\begin{tabular}{lc}
\hline Patient pathologic M stage & \\
\hline 0 & 99 \\
\hline 1 & 1 \\
\hline Tumor histology & 77 \\
\hline Adenocarcinoma & 8 \\
\hline Carcinosarcoma & 4 \\
\hline Clear cell carcinoma & 11 \\
\hline Papillary serous carcinoma & 57 \\
\hline Yes & 41 \\
\hline No & 40 \\
\hline Location of positive nodes & 20
\end{tabular}

or to $0.5 \mathrm{~cm}$. Starting in 2009 , all patients treated with brachytherapy had image simulation. This verification was a quality control measure to account for accuracy of the measured distended vaginal length and proper applicator placement within the pelvis as shown in Figure 1. Most patients received 12-15 Gy in 3 fractions over the course of 7-14 days.

\section{Outcomes}

The median follow up time was 43 months. Among the 100 patients available for disease related outcome

\begin{tabular}{|c|c|}
\hline Tumor characteristics & Patient number \\
\hline \multicolumn{2}{|l|}{ Adnexal involvement } \\
\hline Yes & 30 \\
\hline No & 69 \\
\hline \multicolumn{2}{|l|}{ Parametria involvement } \\
\hline Yes & 15 \\
\hline No & 85 \\
\hline \multicolumn{2}{|l|}{ Vaginal involvement } \\
\hline Yes & 8 \\
\hline No & 92 \\
\hline \multicolumn{2}{|l|}{ Positive pelvic wash } \\
\hline Yes & 12 \\
\hline No & 87 \\
\hline \multicolumn{2}{|l|}{ Tumor grade } \\
\hline 1 & 28 \\
\hline 2 & 26 \\
\hline 3 & 0 \\
\hline \multicolumn{2}{|c|}{ Lower uterine involvement } \\
\hline Yes & 43 \\
\hline No & 57 \\
\hline \multicolumn{2}{|c|}{ Cervical stroma involvement } \\
\hline Yes & 31 \\
\hline No & 69 \\
\hline \multicolumn{2}{|c|}{ Cervical gland involvement } \\
\hline Yes & 35 \\
\hline No & 64 \\
\hline \multicolumn{2}{|l|}{ Patient's margin status } \\
\hline Negative & 92 \\
\hline Close $(<5 \mathrm{~mm})$ & 2 \\
\hline Positive & 5 \\
\hline
\end{tabular}

analysis, 94 patients experienced vaginal control. There was no statistically significant association between vaginal control and a brachytherapy boost $(p=0.51)$. Of those treated with a brachytherapy boost, 82 patients (94\%) experienced vaginal control. Of those who failed in the vagina, 5 of 6 were treated with a VBB. There were no statistically significant differences in vaginal control when examining pathologic variables such as grade, LVSI, CSI, MI, or LUS involvement in the population as shown in Table 3. In 8 patients who had VI, one experienced vaginal failure. There was no statistical difference in rates of vaginal control based on vaginal involvement $(p=0.40)$. 
Ten percent of patients failed in the pelvis as defined by failure in the pelvis and/or vagina, 7 of which were treated with a vaginal brachytherapy boost. For patients who experienced pelvic control, 93\% were treated with a VBB. There was a trend for increasing pelvic control with the use of a boost, $p=0.056$. There was no statistically significant increase in pelvic failure based on LVSI, lymph node status, CSI, VI, or LUS involvement as shown in Table 3. During the follow up period, $11 \%$ recurred with distant metastatic disease, $15 \%$ in the para-aortic or pelvic lymph nodes, $13 \%$ failed in the abdomen.

\section{Discussion}

Adjuvant therapy for locally advanced endometrial cancer continues to evolve. Our study seeks to understand the utility of a VBB, review the existing literature on stage 3 endometrial cancer outcomes, and explore the potential benefit of a VBB on vaginal and pelvic control. There is paucity in the literature regarding the addition of a VBB to whole pelvic radiation in this group of patients, and who may benefit from this additional therapy to whole pelvic radiation.

For patients with stage III endometrial cancer, the attention to pelvic control is relevant. With the publication of GOG 122 in 1995, adjuvant systemic chemotherapy was, in many regards, considered the new standard of care after demonstrating an overall survival benefit of systemic therapy over whole abdominal radiation at 5 years [7]. However, in the systemic therapy group, the risk of relapse in the pelvis neared $20 \%$ [7]. Furthermore, several single institution studies have shown increased rates of pelvic relapse much higher than was demonstrated in GOG 122 [7-10]. In fact, Mundt et al. showed a pelvic recurrence rate of $47 \%$ in high risk pathologic endometrial adenocarcinoma treated with adjuvant chemotherapy and no radiation therapy. In addition, Klopp et al. published their single institutional data confirming a high rate of pelvic failure in those receiving chemotherapy alone [8]. In this series, five-year pelvic-relapse-free survival $(98 \%$ vs. $61 \%, p=0.001)$, DSS $(78 \%$ vs. $39 \%, p=0.01)$, and overall survival ( $73 \%$ vs. $40 \%, p=0.03)$ were significantly better for the regional RT group than the systemic therapy group [8]. Another study by Secord et al. reviewed 256 patients with stage IIIC endometrial cancer [6]. The three-year RFS was $56 \%$ for chemotherapy alone, compared to $73 \%$ for radiation alone, and $73 \%$ for combination therapy $(p=0.12)$. Those receiving chemotherapy alone had the worst 3 -year OS $(78 \%)$ compared to either radiotherapy alone $(95 \%)$, or combination therapy $(90 \%)$ $(p=0.005)$ [6]. They conclude that radiation alone or chemotherapy and radiation was associated with improved outcomes for patients with optimally resected stage IIIC adenocarcinoma compared to those treated with chemotherapy only [6]. Therefore, the risk of vaginal and pelvic control remains an issue in patients treated with chemotherapy alone, arguing for a role of consolidative radiation therapy.

Our study is one of the largest in the literature to review stage III endometrial cancer related outcomes, and the only institutional data specifically investigating the

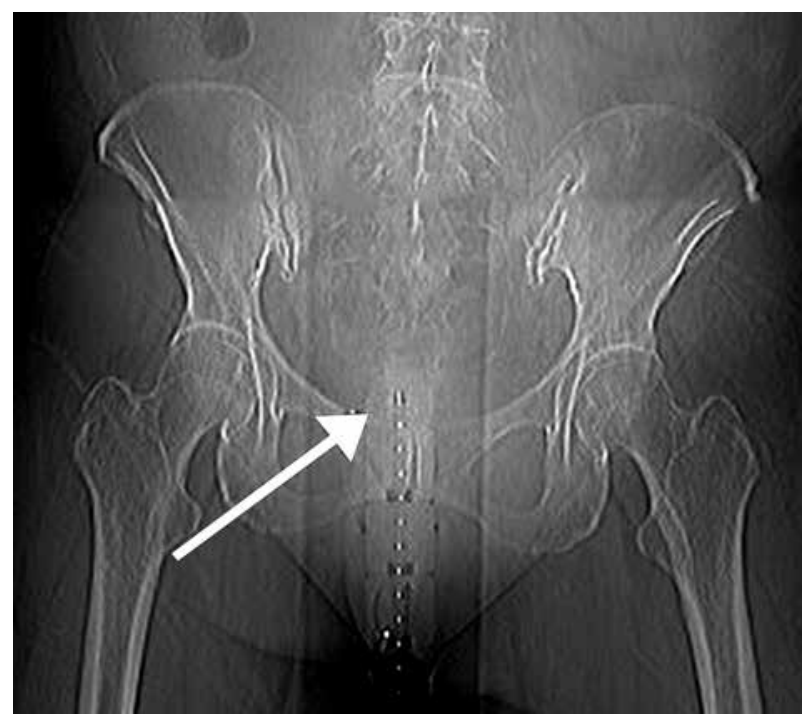

Fig. 1. Image verification of a vaginal brachytherapy implantation

role of a brachytherapy boost in this population. The role of a VBB remains controversial with wide differences in practice pattern variation. In a recent SEER analysis of stage IIIC endometrial adenocarcinoma, 51\% were treated with adjuvant pelvic radiation and $21 \%$ were given a brachytherapy boost [11]. Even in the ongoing cooperative group trials, such as the current GOG 258, which randomizes stage III patients to chemotherapy alone versus chemoradiation followed by systemic therapy, the addition of vaginal brachytherapy is at the discretion of the treating physician. Depending on the instution of treatment, patients are often offered a vaginal brachytherapy boost in the setting of LVSI, high grade disease, VI, or CSI. There also remains debate on the amount of vagina to include in the brachytherapy boost field. The majority of our patients were treated to the upper half of the vagina, based on a vaginal measurement that was performed in the clinic prior to brachytherapy.

One of the major factors in the decision to give a VBB is the potential risk of additional complications. As re-

Table 3. Pathological characteristic variables on peIvic and vaginal control using the Fisher's exact test for significance

\begin{tabular}{lcc} 
Pathologic characteristic & $\begin{array}{c}\text { Pelvic control } \\
p \text {-value }\end{array}$ & $\begin{array}{c}\text { Vaginal control } \\
p \text {-value }\end{array}$ \\
\hline VBB & 0.056 & 0.508 \\
\hline LVSI & 0.462 & 1 \\
\hline Cervical Involvement & 0.495 & 0.370 \\
\hline Grade & 0.691 & 0.759 \\
\hline Lymph node status & 0.915 & 0.764 \\
\hline Vaginal involvement & 0.583 & 0.401 \\
\hline Lower uterine segment & 0.741 & 0.397
\end{tabular}

VBB - vaginal brachytherapy boost, LVSI - lymphovascular space invasion 
ported in the literature, there is an acceptable rate of reported toxicity with the addition of a VBB. Klopp et al. described the major complications in stage 3 endometrial cancer after pelvic radiation with $86 \%$ of patients receiving a VBB [8]. There was one grade 4 small bowel obstruction in a patient treated with external beam RT, for an overall radiation-related major complication rate of $2 \%$ [8]. Rates of minor complications were not recorded [8]. In the RTOG trial 97-08, 22\% of endometrial cancer patients had stage IIIC [12]. The treatment for the 46 patients on trial was surgery followed by adjuvant concurrent chemoradiotherapy. Patients received whole pelvis RT to 45 Gy followed by a VBB, with cisplatin $\left(50 \mathrm{mg} / \mathrm{m}^{2}\right)$ administered on days 1 and 28, followed by four courses of cisplatin $\left(50 \mathrm{mg} / \mathrm{m}^{2}\right)$ and paclitaxel $\left(175 \mathrm{mg} / \mathrm{m}^{2}\right)$ [12] At 4 years, the reported late toxicity for this regimen was grade 1 in $16 \%$, grade 2 in $41 \%$, grade 3 in $16 \%$, and grade 4 in $5 \%$ [12].

In our study, there was a trend for increased pelvic control in those who had VI and were boosted with brachytherapy. Eighty-two of the one hundred patients in the study were treated with a VBB to achieve a vaginal control rate of $94 \%$ and pelvic control rate of $90 \%$. This control rate is impressive given that $57 \%$ had LVSI, 30\% had adenexal involvement, $43 \%$ had LUS involvement, $31 \%$ CSI, and $8 \%$ had VI. In addition, the vast majority of our patients had lymph node involvement. In the SEER analysis, the addition of a radiation therapy improved survival in those with stage IIIC endometrial cancer with direct tumor extenstion with a 5 year overall survival rate of $34 \%, 47 \%$, and $63 \%$ in those receiving adjuvant chemotherapy alone, external beam radiation, and a vaginal brachytherapy boost [11]. Furthermore, when direct extension of the primary tumor was present, the addition of brachytherapy conferred a greater survival advantage [11]. In our population, there was a trend for improved vaginal control in those who had vaginal involvement, but there was no effect of the VBB in respect to margin status. Furthermore, our study population experienced a high vaginal control rate, $94 \%$, and pelvic control, $90 \%$ with the majority of patients treated with trimodality therapy: chemotherapy, consolidative pelvic radiation, and a VBB.

The ongoing question in the field is the questionable incremental benefit of additional radiation to the vaginal apex. Is the addition of vaginal brachytherapy clinically significant for reducing apex recurrences? Should it be given to all patients with stage III endometrial adenocarcinoma? Unfortunately, our data is not able to definitively answer this question. A limitation of our study is that a high number of patients were treated with a brachytherapy boost, reflecting the decision of the physician to deliver a boost in the majority of cases. Therefore, our paper is intended to be thought provoking on the selection and role of a vaginal brachytherapy boost in this defined patient population. Our study reports a low percentage of locoregional failure. Therefore, we are unable to illustrate risk factors that could potentially predict for vaginal failure, and thus warrant the use of a VBB. Perhaps the combination of systemic therapy and radiation therapy are altering the patterns of failure, and the vaginal con- trol would be sufficient without the additional therapy. In terms of patterns of failure, a phase III trial of adjuvant chemotherapy versus pelvic radiotherapy was conducted by Maggi et al. After a median follow-up was 95.5 months, it failed to replicate the results of GOG 122 and revealed no statistical difference in overall survival or progression free survival. The chemotherapy arm trended towards delayed metastatic disease and the radiotherapy arm trended towards improved local control, but neither achieved statistical significance. However, this trial did not report use of vaginal brachytherapy. Compared to single institutional data and the results of GOG 122, our patients had improved vaginal and pelvic control consistent with a recently published retrospective review from Brigham and Women's Hospital, which examined the outcomes of patients treated with adjuvant therapy for FIGO IIIC endometrial adenocarcinoma $[3,4,6,7,9,10]$.

\section{Conclusions}

There is no consensus on the addition of a VBB in stage 3 endometrial carcinoma. The majority of our patients with stage III endometrial cancer were treated with a VBB, and experienced excellent pelvic and vaginal control. There was no difference between pelvic or vaginal control with the addition of a VBB. However, the presence of high grade, VI, LVSI, CSI did not adversely affect outcomes in our patient cohort, suggesting that the role of a VBB in this population needs further exploration.

\section{Acknowledgements}

Cancer Center Support Grant NIH/NCI 5P30CA 093373-10.

\section{Disclosure}

Authors report no conflict of interest.

\section{References}

1. Siegel R, Naishadham D, Jemal A. Cancer statistics, 2012. CA Cancer J Clin 2012; 62: 10-29.

2. Hogberg T, Signorelli M, de Oliveira CF et al. Sequential adjuvant chemotherapy and radiotherapy in endometrial cancer - results from two randomised studies. Eur J Cancer 2010; 46: 2422-2431.

3. Lee LJ, Viswanathan AN. Combined chemotherapy and radiation improves survival for node-positive endometrial cancer. Gynecol Oncol 2012; 127: 32-37.

4. Nelson G, Randall M, Sutton G et al. FIGO stage IIIC endometrial carcinoma with metastases confined to pelvic lymph nodes: analysis of treatment outcomes, prognostic variables, and failure patterns following adjuvant radiation therapy. Gynecol Oncol 1999; 75: 211-214.

5. Schorge JO, Molpus KL, Goodman A et al. The effect of postsurgical therapy on stage III endometrial carcinoma. Gynecol Oncol 1996; 63: 34-39.

6. Secord AA, Geller MA, Broadwater G et al. A multicenter evaluation of adjuvant therapy in women with optimally resected stage IIIC endometrial cancer. Gynecol Oncol 2013; 128: 65-70.

7. Randall ME, Spirtos NM, Dvoretsky P. Whole abdominal radiotherapy versus combination chemotherapy with doxo- 
rubicin and cisplatin in advanced endometrial carcinoma (phase III): Gynecologic Oncology Group Study No. 122. J Natl Cancer Inst Monogr 1995; 19: 13-15.

8. Klopp AH, Jhingran A, Ramondetta L et al. Node-positive adenocarcinoma of the endometrium: outcome and patterns of recurrence with and without external beam irradiation. Gynecol Oncol 2009; 115: 6-11.

9. Mundt AJ, McBride R, Rotmensch J et al. Significant pelvic recurrence in high-risk pathologic stage I-IV endometrial carcinoma patients after adjuvant chemotherapy alone: implications for adjuvant radiation therapy. Int J Radiat Oncol Biol Phys 2001; 50: 1145-1153.

10. Mundt AJ, Murphy KT, Rotmensch J et al. Surgery and postoperative radiation therapy in FIGO Stage IIIC endometrial carcinoma. Int J Radiat Oncol Biol Phys 2001; 50: 1154-1160.

11. Rossi PJ, Jani AB, Horowitz IR et al. Adjuvant brachytherapy removes survival disadvantage of local disease extension in stage IIIC endometrial cancer: a SEER registry analysis. Int J Radiat Oncol Biol Phys 2008; 70: 134-138.

12. Greven $\mathrm{K}$, Winter $\mathrm{K}$, Underhill $\mathrm{K}$ et al. Final analysis of RTOG 9708: Adjuvant postoperative irradiation combined with cisplatin/paclitaxel chemotherapy following surgery for patients with high-risk endometrial cancer. Gynecol Oncol 2006; 103: 155-159. 